\section{USE OF AUTOLOGOUS UMBILICAL ARTERY AND VEIN FOR VASCULAR RECONSTRUCTION IN THE NEWBORN}

\begin{abstract}
Autologous umbilical artery and vein were evaluated as vascular conduits in newborn lambs. Eight newborn lambs were delivered transabdominally under sterile conditions at term. The umbilical artery and vein were dissected from the cord and stored in culture media. On the same day, each lamb underwent bilateral superficial femoral artery transection and reconstruction. Nine arteries were reconstructed with autologous umbilical vein interposition grafts, five with umbilical artery interposition grafts, and two by primary native artery anastomosis. After the birth weight of the lambs quadrupled ( 37 to 45 days), they were killed and all grafts and anastomoses were examined grossly and histologically. At the conclusion of the study, both native artery anastomoses (2/2) were patent. Five umbilical vein (5/9) and two umbilical artery (2/5) autografts were also widely patent. Patent autografts retained an intact endothelium supported by a viable media. The nonpatent autografts had become atrophic remnants displaying histologic signs of early closure. Graft failures are attributed to the extreme vasoactive nature of the umbilical vessels. These preliminary results suggest that umbilical vessels may be useful as a vascular autograft if the vasoactive nature of these vessels can be overcome during the immediate perioperative period. (J THORAC CARDIOvaSC SURG 1995;109:854-7)
\end{abstract}

Angelo A. Vlessis, MD, PhD, ${ }^{a}$ Hagop Hovaguimian, MD, ${ }^{\text {a, b }}$ Eric Arntson, MD, and Albert Starr, MD, ${ }^{\mathrm{b}}$ Portland, Ore. $\bigvee_{\mathrm{e}, \mathrm{s}}^{\mathrm{a}}$ ascular substitutes, such as polytetrafluoroethylene, Dacron polyester, and treated pericardium, play an integral role in the surgical repair of many congenital cardiovascular defects. Unfortunately, several studies document an increased incidence of endocarditis in patients whose repairs involved synthetic materials. ${ }^{1-3}$ In addition, nonviable grafting materials do not enlarge as the child grows. Subsequent reoperation(s) may become necessary to improve hemodynamics in the area of the graft as the patient grows. ${ }^{4}$ Although synthetic and nonviable vascular substitutes have enhanced the successful repair of many congenital cardiovascular defects, the optimal grafting material has yet to be described.

Naturally, the ideal intravascular graft would be living, autologous vasculature. An intact endothelium would provide a nonthrombogenic intravascu-

\footnotetext{
From the Oregon Health Sciences University ${ }^{\mathrm{a}}$ and St. Vincent's Hospital and Medical Center, ${ }^{\mathrm{b}}$ Portland, Ore.

Received for publication April 1, 1994.

Accepted for publication Sept. 30, 1994.

Address for reprints: Angelo A. Vlessis, MD, PhD, Department of Surgery, L223A, Oregon Health Sciences University, 3181 S.W. Sam Jackson Park Rd., Portland, OR 97201.

Copyright (C) 1995 by Mosby-Year Book, Inc.

$0022-5223 / 95 \$ 3.00+0 \quad \mathbf{1 2 / 1 / 6 1 0 2 6}$
}

lar surface resistant to bacterial adherence. The intima would be supported by a living media and adventitia that could grow as the surrounding tissues and child grow.

Current advancements in echocardiographic techniques now permit the accurate antenatal diagnosis of many congenital heart defects necessitating surgical palliation or repair, ${ }^{5,6}$ With this prenatal insight, we hypothesized that the autologous cord vessels, if properly preserved, may provide a more suitable material for subsequent vascular reconstruction than those currently used.

Several questions regarding the state of differentiation and the growth potential of the umbilical vasculature remain unanswered. First, is the umbilical vasculature senescent and doomed to atrophy or will this tissue remain viable if autografted into physiologically similar milieu? Second, are the cells of the umbilical vasculature fully differentiated and incapable of growth or does this tissue have further growth potential? With these questions in mind, we explored the utility of autologous umbilical artery and vein as an arterial substitute in the newborn lamb.

\section{Materials and methods}

Experimental animals. Four pregnant ewes carrying twins were obtained near term. Lambs were delivered 

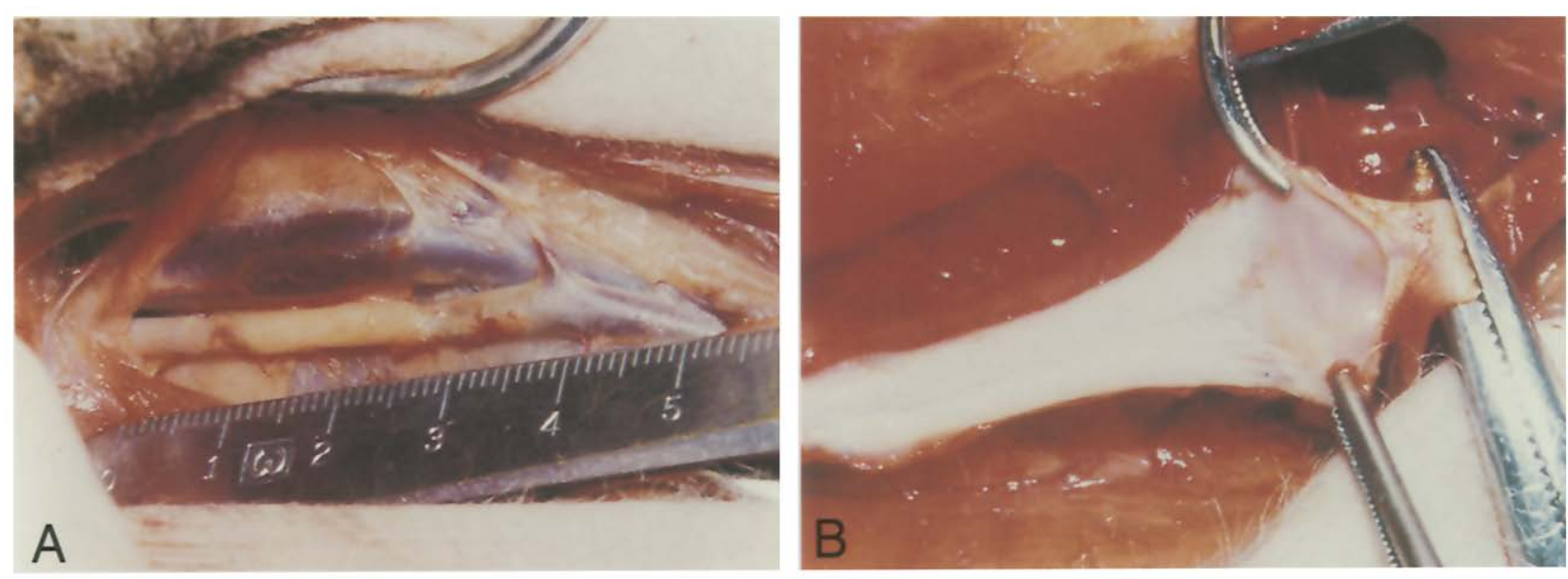

Fig. 1. A, Umbilical artery autograft in situ at necropsy. The proximal native vessel is to the right. $\mathbf{B}$, Autograft of $\mathbf{A}$ after the graft was opened longitudinally. The inner surface of the native artery and autograft are covered by a smooth intimal surface. No evidence of stenosis or hyperplasia exists grossly. Note that the midportion of the graft is in spasm and, as a consequence, appears narrowed.

transabdominally under sterile conditions. The autologous umbilical vessels were properly labeled, dissected free from the cord, and placed in sterile culture media until implantation. In contrast to the human condition, the lamb umbilical cord has two veins and two arteries. Once these vessels are manipulated, the distinction between artery and vein becomes very difficult because of spasm. Proper identification was facilitated by labeling the veins on the intact cord before transection.

Eight viable lambs were delivered, yielding sixteen superficial femoral arteries for grafting experiments. All lambs underwent operation (see Operation) on the delivery date and were recovered without complication. Lambs received aspirin $(20 \mathrm{mg})$ every day starting the afternoon of operation. Once lambs achieved four times their birth weight ( 37 to 45 days), they were killed by intravenous injection of thiopental sodium (Pentothal). The umbilical autografts and anastomoses were then examined both grossly and histologically.

All procedures were approved and supervised by the Animal Care Department at Oregon Health Sciences University in compliance with state and federal regulations.

Operation. Under general anesthesia, each lamb had both superficial femoral arteries exposed. Animals were given heparin (100 units $/ \mathrm{kg}$ ) and arterial anastomoses were performed in an end-to-end tapered fashion with a running 8-0 nonabsorbable suture. Two arteries underwent simple transection and reanastomosis and the other fourteen arteries underwent transection with umbilical vessel interposition grafting. Five umbilical artery and nine umbilical vein autografts were placed. All autografts were open at the conclusion of the operation except for two umbilical artery grafts, which remained in complete vasospasm despite topical papaverine and mechanical dilation.
Histology. Excised specimens were immediately placed in $10 \%$ buffered formalin. Sections were prepared for examination with a light microscope after being stained with hematoxylin and eosin. Transverse sections through the center of each autograft, as well as longitudinal sections through each anastomosis, were examined by a pathologist (E.A.). Proper identification of umbilical artery versus vein was confirmed by the presence or absence of vasa vasorum, respectively, as well as muscular wall thickness and luminal size.

\section{Results}

The two superficial femoral arteries in which simple transection and reanastomosis were performed were widely patent by gross examination. Histologically, significant intimal hyperplasia on both the proximal and distal sides of the anastomosis was evident as a proliferation of the intima on the luminal side of the internal elastic lamina.

Five umbilical artery interposition autografts were placed. Two of these failed immediately at operation because of extreme vasospasm in the autograft. Of the remaining three umbilical artery autografts, two were patent at the conclusion of the study by gross examination (Fig. 1). The luminal diameter of the native vessel enlarged from approximately $3 \mathrm{~mm}$ to $5 \mathrm{~mm}$ between birth and necropsy in animals with patent autografts. Histologically, the patent autografts showed minimal to no intimal hyperplasia, and all components of the vessel wall remained intact. The occluded umbilical 

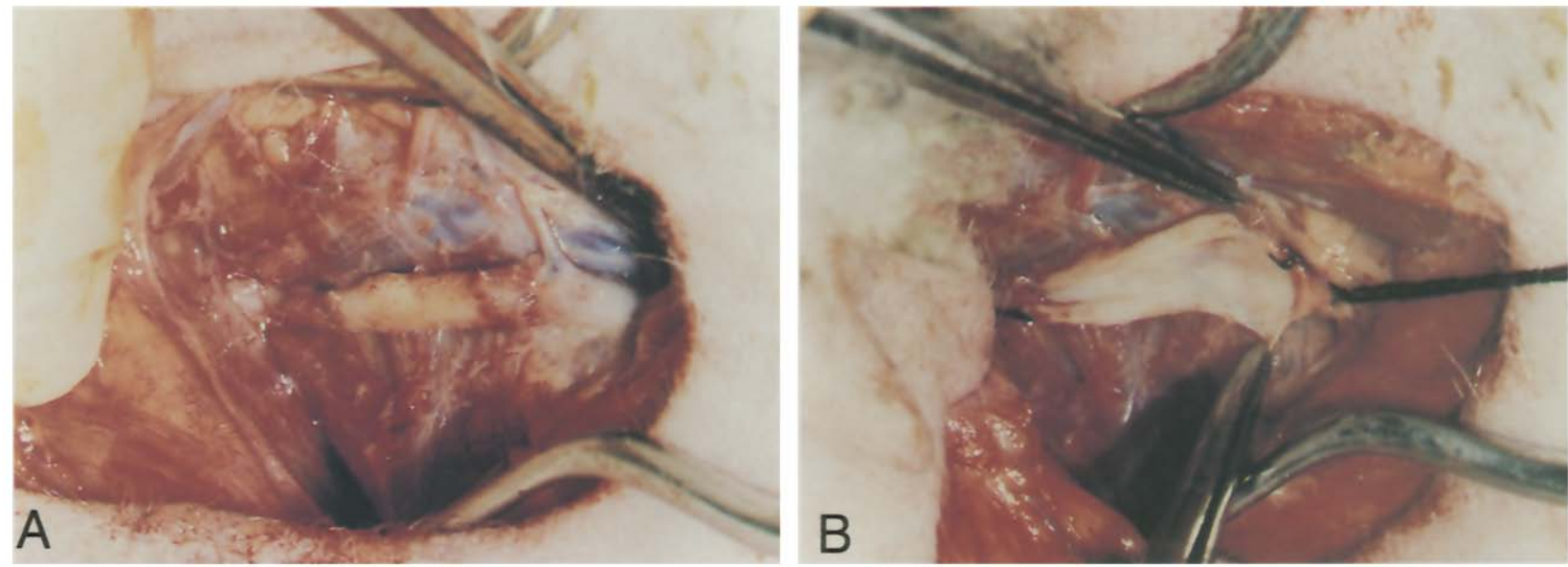

Fig. 2. A, Patent umbilical vein autograft in situ at necropsy. The proximal native artery is to the left. B, Vein autograft of $\mathbf{A}$ after the graft was opened longitudinally. The luminal surface is smooth and shiny without stenosis. This autograft is supple and pliant without the spasticity encountered in the arterial autografts.

artery autografts were small cordlike structures in situ. The proximal and distal native vessels were occluded. Collateral vessels were prominent. Histologically, the lumina were filled with hyperplastic intima and the media appeared atrophic. The intima and media were distinguishable with difficulty inasmuch as the internal elastic lamina was obliterated. No signs of inflammation were evident in any of the specimens.

Of the nine umbilical vein autografts, only five remained patent at the conclusion of the study. Although difficulties with vasospasm were encountered at operation, all grafts were widely patent before the skin incisions were closed. At necropsy, the patent vein autografts were accompanied by a prominent proximal and distal native artery approximately $5 \mathrm{~mm}$ in diameter (Fig. 2). The lumina were smooth and without evidence of stenosis. Histologically, minimal to no intimal hyperplasia was seen in the midportion of the graft. All components of the vessel wall were intact and without signs of inflammation. Focal nonobstructive intimal hyperplasia was noted at the suture lines. Scattered areas of regional calcification were also noted in the outer media and adventitia. The occluded vein autograft remnants were small and difficult to distinguish from surrounding scar tissue. The adjacent proximal and distal native arteries were small and cordlike. Collateral vessel formation was pronounced. Histologically, a hyperplastic intima and atrophic media remained identifiable. The histologic findings were similar to those seen in segments of obliterated umbilical artery graft. Early recanalization was apparent with several small blood-filled channels seen on transverse sections.

\section{Discussion}

The human extracorporeal umbilical vein is a sizable vessel with a luminal diameter of 4 to $6 \mathrm{~mm}$ and a length of 25 to $140 \mathrm{~cm}^{7}$ This vessel is usually discarded at birth with the placenta. In cases of antenatally diagnosed congenital cardiovascular defects, we postulated that the autologous umbilical vein may be used for the subsequent vascular reconstruction rather than the nonliving alternatives currently used. A literature search was performed and no previous studies describing umbilical vessel autografts in newborn human beings or animals were identified. To our knowledge, this is the first study describing the use of autologous umbilical artery and vein for vascular reconstruction in the newborn.

The results herein describe our preliminary findings in an animal model. Overall, umbilical vessel autograft patency was poor, with only seven of fourteen vessels patent 37 to 45 days after implantation. We attribute the graft failures to the extreme vasospastic nature of these vessels. Two vessels failed immediately as a result of severe spasm. The time of closure for the remaining nonpatent vessels is unknown; however, the marked collateral vessel formation combined with their histologic appearance suggests that these vessels occluded early after 
operation. In retrospect, daily aspirin therapy (see Materials and methods) may have actually promoted autograft closure by inhibiting synthesis of vasorelaxant prostaglandins within the autograft. ${ }^{8}$

A promising feature of this study was the gross and histologic appearance of the autografts that remained patent. These autografts clearly supported the proximal and distal circulation as evidenced by the enlargement of the native vessels and lack of collateral vessel development (Figs. 1 and 2). A single layer of endothelium covered the luminal surface; a viable media supported the intima. These findings demonstrate that the autologous umbilical vasculature can function as a living arterial substitute. If the vessel lumen remains patent, the viability and integrity of the vessel is preserved.

Some calcific changes were seen in the outer segments of the umbilical vein autografts that remained patent. As mentioned earlier, umbilical artery and vein are distinguished by the presence and absence of vasa vasorum, respectively. Therefore the calcific changes seen in the vein autografts may be due to ischemic necrosis and subsequent calcification of the outer portions of the vessel, which do not receive an adequate nutrient supply after implantation.

The umbilical vasculature is derived from the embryo and, as such, is truly autologous. Therefore it is no surprise that histologic evidence of inflammation or rejection was not observed. Furthermore, the umbilical vasculature is not senescent; it has the capability of remaining viable when used as a vascular conduit. More elaborate studies are needed to determine whether the umbilical vasculature has potential for further growth.
Obviously, additional research is needed to determine the clinical utility of autologous umbilical vessels as a practical vascular substitute. Their use as a vascular patch or baffling material also remains to be explored.

\section{REFERENCES}

1. Blumenthal S, Griffiths SP, Morgan BC. Bacterial endocarditis in children with congenital heart disease. Pediatrics 1960;26:993-1017.

2. Geva T, Frand M. Infective endocarditis in children with congenital heart disease: the changing spectrum 1965-1985. Eur Heart J 1988;9:1244-9.

3. Awadallah SM, Kavey RW, Byrum CJ, Smith FC, Kveselis DA, Blackman MS. The changing pattern of infective endocarditis in childhood. Am J Cardiol 1991; 68:90-4.

4. Fernandez G, Costa F, Fontan F, Naftel DC, Blackstone EH, Kirklin JW. Prevalence of reoperation for pathway obstruction after Fontan operation. Ann Thorac Surg 1989;48:654-9.

5. Cullen S, Sharland GK, Allan LD, Sullivan ID. Potential impact of population screening for prenatal diagnosis of congenital heart disease. Arch Dis Child 1992;67:775-8.

6. Shenker L, Reed KL, Marx GR, Donnerstein RL, Allen HD, Anderson CF. Fetal cardiac Doppler flow studies in prenatal diagnosis of heart disease. Am J Obstet Gynecol 1988;158:1267-73.

7. Malpas P. Length of human umbilical cord at term. $\mathrm{Br}$ Med J 1964;1:673-4.

8. Paulick RP, Meyers RL, Rudolph CD, Rudolph AM. Venous and hepatic vascular responses to indomethacin and prostaglandin $E_{1}$ in the fetal lamb. Am J Obstet Gynecol 1990;163:1357-63. 